\title{
Molecular Dissection of $A R P 1$ Regions Required for Nuclear Migration and Cell Wall Integrity Checkpoint Functions in Saccharomyces cerevisiae
}

\author{
Ryoji Igarashi, Masaya Suzuki, Satoru Nogami, and Yoshikazu Ohya* \\ Laboratory of Signal Transduction, Department of Integrated Biosciences, Graduate School of Frontier \\ Sciences, University of Tokyo, 5-1-5 Kashiwanoha, Kashiwa, Chiba 277-8562, Japan
}

\begin{abstract}
The dynactin complex is one of the components required for the regulation of the cell wall integrity checkpoint, which ensures the completion of cell wall remodeling before mitosis. The core of the dynactin complex is a backbone filament composed of monomers of an actin-related protein, Arp1, which is also involved in nuclear migration. To examine the molecular basis for the dual functions of the dynactin core subunit Arp1p in yeast, we constructed 32 mutated arpl alleles. We assessed the effects of the mutations on cell wall integrity checkpoint and nuclear migration functions and identified four categories of mutants: 1) those showing no change from the wild type; 2) those resulting in a defective cell wall integrity checkpoint but normal nuclear migration; 3) those with a normal cell wall integrity checkpoint but defective nuclear migration; and 4) those defective in both the cell wall integrity checkpoint and nuclear migration functions. Our results show a separation of the two functions in the molecular structure of Arp1p and indicate that a local surface region of Arp1p is important in maintaining the cell wall integrity checkpoint function.
\end{abstract}

Key words: cell wall integrity checkpoint/dynactin complex/ARP1/alanine scanning/nuclear migration/1,3- $\beta$-glucan synthase

\section{Introduction}

Cell cycle progression is regulated by several checkpoint mechanisms that monitor cell cycle landmarks. When a cell cycle event is perturbed, progression of the cell cycle to subsequent events is halted until the completion of the former event. Well-known checkpoint targets are DNA damage, DNA replication (Weinert et al., 1994), kinetochore attachment to the mitotic spindle (Rudner and Murray, 1996), and bud morphogenesis (Lew and Reed, 1995).

We recently discovered a new cell cycle checkpoint, termed the cell wall integrity checkpoint (Suzuki et al., 2004). In response to a defect in cell wall synthesis, cells of the yeast Saccharomyces cerevisiae arrest cell growth at the small bud before entry into mitosis. The cell wall of $S$. cerevisiae consists predominantly of $1,3-\beta$-glucan (Cid et al., 1995). Mutants defective in 1,3- $\beta$-glucan synthesis cannot form the medium or large bud, and these mutants

\footnotetext{
*To whom correspondence should be addressed: Yoshikazu Ohya, Laboratory of Signal Transduction, Department of Integrated Biosciences, Graduate School of Frontier Sciences, University of Tokyo, 5-1-5 Kashiwanoha, Kashiwa, Chiba 277-8562, Japan.

Tel: +81-4-7136-3650, Fax: +81-4-7136-3651

E-mail: ohya@k.u-tokyo.ac.jp
}

arrest the cell cycle progression before nuclear division without forming a bipolar spindle (Suzuki et al., 2004). This observation indicates that the perturbation of $1,3-\beta$-glucan synthesis triggers the cell wall integrity checkpoint, leading to cell cycle arrest before the separation of the spindle pole body (SPB). An analysis of the mutant defective in the cell wall integrity checkpoint, wacl (wall checkpoint defective), revealed that this mutant accumulates cells with a bipolar spindle after 1,3- $\beta$-glucan synthesis has stopped (Suzuki et al., 2004). WAC1 is identical to $A R P 1$, and the wacl product possesses a single amino acid replacement (P268L). These findings suggest that Arp1p (Waclp) is required to arrest the cell cycle at G2 phase when cell wall synthesis is perturbed, but little is known about how Arp1p actually functions in the cell wall integrity checkpoint.

Biochemical and microscopic studies have revealed that Arp1p is the core subunit of the dynactin complex. Nine or 10 Arp1p monomers form the actin-like short filament backbone of the dynactin complex, as well as multiple species of subunits assemble along this filament (Eckley et al., 1999; Schafer et al., 1994). The dynactin complex of the budding yeast contains at least three species of polypeptides: Arp1p, Nip100p, and Jnm1p (Geiser et al., 1997; Kahana et al., 1998; McMillan and Tatchell, 1994). In addition to these proteins, comprehensive two-hybrid screening 
has isolated three Arp1p binding proteins: Arp10p, Jsn1p, and Yjr008wp (Ito et al., 2001; Uetz et al., 2000). Genetic studies have shown that the dynactin complex is required for the nuclear migration process after anaphase. The deletion of the ARP1 gene causes the defect in dynein-mediated nuclear migration, and cells with two nuclei in the mother cell are frequently observed (Muhua et al., 1994). The dynactin complex in $S$. cerevisiae is proposed to associate with the cytoplasmic dynein motor, resulting in the pulling force to promote nuclear migration (Hildebrandt and Hoyt, 2000). In addition, components of the dynactin complex, namely Arp1p, Nip100p, and Jnm1p, are required for the cell wall integrity checkpoint. Surprisingly, the dynactinrelated proteins Dyn1p, Pac11p, Num1p, Pac1p, and Bik1p are not required for the checkpoint (Suzuki et al., 2004). Thus Arp1p functions in the cell wall integrity checkpoint as well as in nuclear migration, although the relationship between these two functions remains unclear.

In this study, we used a genetic approach to determine whether the cell wall integrity checkpoint and nuclear migration functions of Arp1p are based on independent molecular functions. We constructed 32 mutated arp1 alleles with alanine mutations at the clusters of charged amino acids that are predicted to be on the surface of the Arp1p ultrastructure. We then examined the effects of the mutations on the cell wall integrity checkpoint and nuclear migration functions of Arp1p in yeast cells.

\section{Materials and Methods}

\section{Strains and plasmids}

Standard molecular biological techniques for yeast and bacteria were used (Sambrook et al., 1989; Sherman et al., 1986).

The yeast strains used in this study are listed in Table I. All strains are derivatives of YPH499 and YPH500 (Sikorski and Hieter, 1989). The FKS1 or $f k s 1-1154$ mutant strains used in this study harbored an FKS1 or $f k s 1-1154$ temperature-sensitive allele integrated into the $\Delta f k s 1 \Delta f k s 2$ background, in order to avoid the effect of Fks2p that has overlapping functions with Fks1p. The FKS1 wacl (YOC2857) and fks1-1154 wacl (YOC2858) strains have the wacl mutation (arp1 ${ }^{\mathrm{P} 268 \mathrm{~L}}$ ) in ARP1 of the FKS1 (YOC1001) and $f k s 1-1154$ (YOC1087) strains, respectively.

The genomic ARP1 was replaced with the gene Cg-LEU2 (Candida glablata LEU2 gene compatible with Saccharomyces cerevisiae LEU2) in strains FKS1 and fks 1-1154 to generate the FKS1 $\Delta a r p 1$ (YOC2888) and fks1-1154 $\Delta a r p 1$ (YOC2889) strains, respectively.

The arp1-x:URA3 strains (YOC3032-3099) were constructed by replacing the $\triangle$ arp $1:: L E U 2$ locus of the FKS1 $\triangle$ arp 1 (YOC2888)

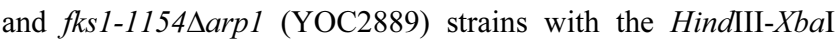
fragment containing arp 1-x:URA3 prepared from the pBS-arp1-x plasmid. In the wacl:URA3 strains, the genomic $\triangle$ arp $1: \because L E U 2$ region was replaced with a HindIII-DraI fragment, because wacl was digested with $X b a I$. The integrants were selected on SD-uracil plates, and the correct integrations were verified by PCR.

The YOC4106-4112 were constructed by transforming the plasmid pYO2763, centromeric vector containing $K A R 9$ and $U R A 3$, into FKS1 (YOC1001), FKS1 wac1 (YOC2857), FKS1 $\triangle a r p 1::$ KanMX (YOC4113), FKS arp1-31 (YOC3035), FKS1 arp1-236 (YOC3052), FKS1 arp1-266 (YOC3056) and FKS1 arp1-374 (YOC3065) strains (URA3 marker of genomic YOC3035, 3052, 3056 and 3065 were deleted). The genomic KAR9 gene of the resultant strains was then replaced with the $C g-L E U 2$ marker.

The plasmids used in this study are listed in Table II. The plasmid pRS315-ARP1 (pYO2521) contains the 2.1-kb HindIII$X b a \mathrm{I}$ fragment, including the entire ORF of $A R P 1$, inserted into pRS315. For pBS-ARP1-URA3 (pYO2522), the 2.1-kb HindIII$X b a \mathrm{I}$ fragment, including the entire ORF of $A R P 1$, was prepared from pRS315-ARP1 and was inserted into pBluescript II SK(+). The yeast URA3 fragment amplified from pRS316 by PCR was digested with $S m a I$ and was inserted at the blunt-ended EcoT22I site located $200 \mathrm{bp}$ upstream from the $X b a \mathrm{I}$ site.

For pBS-wac1-URA3 (pYO2523), a fragment including the wacl allele was amplified from the FKS1 wacl genome by PCR, digested with HindIII and DraI, and used to replace the HindIIIDraI region of pBS-ARP1-URA3. Positive clones were selected based on $X b a I$ digestion, because an additional $X b a I$ site was generated by the wacl mutation.

To construct pBS-arp1-x-URA3 (pYO2524-pYO2555), the NruISphI fragment of pBS-ARP1-URA3 (or, in the case of arp1-294 to -374, the SphI-EcoT22I fragment) was replaced with EGFP. Next, the EGFP fragment inserted in pBS-ARP1-URA3 was replaced with the NruI-SphI fragment (or, in the case of arp1-294-arp1374 , the SphI-EcoT22I fragment), which included a part of the ORF of arp 1-x containing an alanine mutation, prepared from mutated pRS315-ARP1. Positive clones were selected based on plasmid digestion with HindIII and Bsp1407I.

\section{Site-directed mutagenesis}

A QuickChange site-directed mutagenesis kit (Stratagene, La Jolla, CA, USA) was used to make point mutations. We used 32 synthetic oligonucleotides to change selected charged amino acids in yeast $A R P 1$ to alanine. The designed mutations were placed in the middle of each oligonucleotide, with 15-20 bases of correct sequence in each flanking region. Using pRS315-ARP1, which included intact $A R P 1$ as a template, the mutagenic oligonucleotide primers complementary to opposite strands of the plasmid were extended by PCR. Following the PCR, the products were treated with $D p n$ I (target sequence: $5^{1}-\mathrm{Gm}^{6} \mathrm{ATC}-3^{\prime}$ ), which is specific for methylated and hemimethylated DNA, to digest the parental DNA template and to select for the mutation-containing synthesized DNA. Template plasmid isolated from Escherichia coli is methylated and therefore susceptible to DpnI digestion. The nicked plasmids incorporating the desired mutation were then transformed into E. coli XL-1-Blue, and the mutations were confirmed by DNA sequencing. 
Table I. YeAST STRAINS USED IN THIS STUDY

\begin{tabular}{|c|c|}
\hline Strains & Genotype \\
\hline YOC1001 (FKS1) & MATa $f k s 1:: H I S 3$, fks $2:: L Y S 2$, ade $3:: F K S 1: T R P 1$ \\
\hline YOC2857 (FKS1 wacl) & MATa fks $1:: H I S 3$, fks $2: \because L Y S 2$, ade $3:: F K S 1: T R P 1$, wac1 \\
\hline YOC2888 (FKS1 $\Delta a r p l)$ & MATa $f k s 1: \because H I S 3, f k s 2:: L Y S 2$, ade $\because: F K S 1: T R P 1$, arp $1: C g-L E U 2 *$ \\
\hline YOC4113 (FKS1 $\Delta a r p 1: \because K a n M X)$ & MATa $f k s 1: \because H I S 3$, fks $2:: L Y S 2$, ade $3: \because F K S 1: T R P 1$, arp $1: \because \operatorname{KanMX}$ \\
\hline YOC3032 (FKS1 ARP1:URA3) & MATa fks $1: \because H I S 3$, fks $2: \because L Y S 2$, ade $3: \because F K S 1: T R P 1$, arp $1: \because A R P 1: U R A 3$ \\
\hline YOC3033 (FKS1 wac1:URA3) & MATa fks $1: \because H I S 3$, fks $2:: L Y S 2$, ade $3: \because F K S 1: T R P 1$, arp $1: \because$ wac $1: U R A 3$ \\
\hline YOC3034 (FKS1 arpl-2) & MATa $f k s 1: \because H I S 3, f k s 2: \because L Y S 2$, ade $3: \because F K S 1: T R P 1$, arp $1: \because \operatorname{arp} 1-2: U R A 3$ \\
\hline YOC3035 (FKS1 arp1-31) & MATa $f k s 1: \because H I S 3, f k s 2: \because L Y S 2$, ade $3: \because F K S 1: T R P 1$, arp $1: \because \operatorname{arp} 1-31: U R A 3$ \\
\hline YOC3036 (FKS1 arp1-35) & MATa $f k s 1: \because H I S 3, f k s 2: \because L Y S 2$, ade $3: \because F K S 1: T R P 1$, arp $1: \because \operatorname{arp} 1-35: U R A 3$ \\
\hline YOC3037 (FKS1 arp1-46) & MATa $f k s 1: \because H I S 3, f k s 2: \because L Y S 2$, ade $3: \because F K S 1: T R P 1$, arp $1: \because \operatorname{arp} 1-46: U R A 3$ \\
\hline YOC3038 (FKS1 arp1-67) & MATa $f k s 1: \because H I S 3, f k s 2: \because L Y S 2$, ade $3: \because F K S 1: T R P 1$, arp $1: \because \operatorname{arp} 1-67: U R A 3$ \\
\hline YOC3039 (FKS1 arp1-73) & MATa $f k s 1:: H I S 3, f k s 2:: L Y S 2$, ade3::FKS1:TRP1, arp1::arp1-73:URA3 \\
\hline YOC3040 (FKS1 arp1-79) & MATa $f k s 1:: H I S 3, f k s 2:: L Y S 2$, ade3::FKS1:TRP1, arp1::arp1-79:URA3 \\
\hline YOC3041 (FKS1 arp1-84) & MATa $f k s 1:: H I S 3, f k s 2:: L Y S 2$, ade $3:: F K S 1: T R P 1$, arp $1:: \operatorname{arp} 1-84: U R A 3$ \\
\hline YOC3042 (FKS1 arp1-108) & MATa $f k s 1:: H I S 3$, fks $2 \because: L Y S 2$, ade $3:: F K S 1: T R P 1$, arp $1:$ arp 1-108:URA3 \\
\hline YOC3043 (FKS1 arp1-122) & MATa $f k s 1: \because H I S 3, f k s 2 \because: L Y S 2$, ade $3 \because$ FKS1:TRP1, arp $1: \because \operatorname{arp} 1-122: U R A 3$ \\
\hline YOC3044 (FKS1 arp1-133) & MATa $f k s 1: \because H I S 3, f k s 2: \because L Y S 2$, ade $3:: F K S 1: T R P 1$, arp $1: \because \operatorname{arp} 1-133: U R A 3$ \\
\hline YOC3045 (FKS1 arp1-162) & MATa $f k s 1: \because H I S 3, f k s 2: \because L Y S 2$, ade $3:: F K S 1: T R P 1, \operatorname{arp} 1: \because \operatorname{arp} 1-162: U R A 3$ \\
\hline YOC3046 (FKS1 arp1-185) & MATa $f k s 1: \because H I S 3, f k s 2: \because L Y S 2$, ade $3:: F K S 1: T R P 1$, arp $1: \because \operatorname{arp} 1-185: U R A 3$ \\
\hline YOC3047 (FKS1 arp1-192) & MATa $f k s 1: \because H I S 3$, fks $2: \because L Y S 2$, ade $3:: F K S 1: T R P 1$, arp $1: \because \operatorname{arp} 1-192: U R A 3$ \\
\hline YOC3048 (FKS1 arp1-202) & MATa $f k s 1:: H I S 3$, fks $2:: L Y S 2$, ade3::FKS1:TRP1, arp $1:$ arp 1-202:URA3 \\
\hline YOC3049 (FKS1 arp1-214) & MATa $f k s 1: \because H I S 3, f k s 2 \because: L Y S 2$, ade $3: \because F K S 1: T R P 1$, arp $1: \because \operatorname{arp} 1-214: U R A 3$ \\
\hline YOC3050 (FKS1 arp1-222) & MATa $f k s 1:: H I S 3, f k s 2:: L Y S 2$, ade $3:: F K S 1: T R P 1$, arp $1: \operatorname{arp} 1-222: U R A 3$ \\
\hline YOC3051 (FKS1 arp1-233) & MATa $f k s 1: \because H I S 3$, fks $2:: L Y S 2$, ade3::FKS1:TRP1, arp $1:$ arp 1-233:URA3 \\
\hline YOC3052 (FKS1 arp1-236) & MATa $f k s 1: \because H I S 3$, fks $2:: L Y S 2$, ade $3:: F K S 1: T R P 1$, arp $1:$ arp 1-236:URA3 \\
\hline YOC3053 (FKS1 arp1-251) & MATa $f k s 1: \because H I S 3, f k s 2: \because L Y S 2$, ade $3: \because F K S 1: T R P 1$, arp $1: \because \operatorname{arp} 1-251: U R A 3$ \\
\hline YOC3054 (FKS1 arp1-256) & MATa $f k s 1: \because H I S 3, f k s 2: \because L Y S 2$, ade $3:: F K S 1: T R P 1$, arp $1: \because \operatorname{arp} 1-256: U R A 3$ \\
\hline YOC3055 (FKS1 arpl-263) & MATa $f k s 1: \because H I S 3$, fks $2 \because: L Y S 2$, ade $3:: F K S 1: T R P 1, \operatorname{arp} 1: \because \operatorname{arp} 1-263: U R A 3$ \\
\hline YOC3056 (FKS1 arp1-266) & MATa $f k s 1: \because H I S 3, f k s 2: \because L Y S 2$, ade $3:: F K S 1: T R P 1, \operatorname{arp} 1: \because \operatorname{arp} 1-266: U R A 3$ \\
\hline YOC3057 (FKS1 arp1-282) & MATa $f k s 1: \because H I S 3$, fks $2: \because L Y S 2$, ade $3:: F K S 1: T R P 1$, arp $1: \because \operatorname{arp} 1-282: U R A 3$ \\
\hline YOC3058 (FKS1 arp1-294) & MATa fks $1: \because H I S 3$, fks $2: \because L Y S 2$, ade $3: \because F K S 1: T R P 1$, arp $1: \because \operatorname{arp} 1-294: U R A 3$ \\
\hline YOC3059 (FKS1 arpl-300) & MATa $f k s 1:: H I S 3$, fks $2 \because: L Y S 2$, ade $3:: F K S 1: T R P 1$, arp $1:$ arp $1-300: U R A 3$ \\
\hline YOC3060 (FKS1 arpl-317) & MATa $f k s 1: \because H I S 3$, fks $2:: L Y S 2$, ade3::FKS1:TRP1, arp $1:$ arp 1-317:URA3 \\
\hline YOC3061 (FKS1 arpl-326) & MATa $f k s 1: \because H I S 3, f k s 2:: L Y S 2$, ade3::FKS1:TRP1, arp $1:$ arp 1-326:URA3 \\
\hline YOC3062 (FKS1 arp1-336) & MATa $f k s 1: \because H I S 3, f k s 2:: L Y S 2$, ade $3:: F K S 1: T R P 1$, arp $1: \because \operatorname{arp} 1-336: U R A 3$ \\
\hline YOC3063 (FKS1 arp1-344) & MATa $f k s 1: \because H I S 3, f k s 2:: L Y S 2$, ade $3:: F K S 1: T R P 1$, arp $1: \because \operatorname{arp} 1-344: U R A 3$ \\
\hline YOC3064 (FKS1 arp1-368) & MATa $f k s 1: \because H I S 3$, fks $2: \because L Y S 2$, ade $3:: F K S 1: T R P 1$, arp $1: \because \operatorname{arp} 1-368: U R A 3$ \\
\hline YOC3065 (FKS1 arp1-374) & MATa $f k s 1: \because H I S 3$, fks $2: \because L Y S 2$, ade $3:: F K S 1: T R P 1$, arp $1: \because \operatorname{arp} 1-374: U R A 3$ \\
\hline YOC1087 (fks 1-1154) & MATa fks $1:: H I S 3$, fks $2:: L Y S 2$, ade $3:: f k s 1-1154: T R P 1$ \\
\hline YOC2858 (fks $1-1154$ wacl) & MATa $f k s 1: \because H I S 3$, fks $2:: L Y S 2$, ade $3:: f k s 1-1154: T R P 1$, wac1 \\
\hline YOC2889 (fks 1-1154 $\Delta a r p 1)$ & MATa fks $1: \because H I S 3$, fks $2:: L Y S 2$, ade $3: f k s 1-1154: T R P 1$, arp $1: C g-L E U 2$ \\
\hline YOC3066 (fks 1-1154 ARP1:URA) & MATa fks $1: \because H I S 3$, fks $2 \because: L Y S 2$, ade $3: f k s 1-1154: T R P 1$, arp $1: A R P 1: U R A 3$ \\
\hline YOC3067 (fks 1-1154 wac1:URA) & MATa fks $1: \because H I S 3$, fks $2:: L Y S 2$, ade $3:: f k s 1-1154: T R P 1$, arp $1::$ wac1:URA3 \\
\hline YOC3068 (fks1-1154 arp1-2) & MATa $f k s 1: \because H I S 3, f k s 2: \because L Y S 2$, ade $\because: f k s 1-1154: T R P 1$, arp $1: \because \operatorname{arp} 1-2: U R A 3$ \\
\hline YOC3069 (fks $1-1154 \operatorname{arp} 1-31)$ & MATa $f k s 1: \because H I S 3, f k s 2: \because L Y S 2$, ade3::fks 1-1154:TRP1, arp1::arp1-31:URA3 \\
\hline YOC3070 (fks $1-1154$ arp 1-35) & MATa $f k s 1: \because H I S 3, f k s 2: \because L Y S 2$, ade3::fks 1-1154:TRP1, arp1::arp1-35:URA3 \\
\hline YOC3071 (fks 1-1154 arp1-46) & MATa $f k s 1: \because H I S 3, f k s 2: \because L Y S 2$, ade3::fks 1-1154:TRP1, arp1::arp1-46:URA3 \\
\hline YOC3072 (fks 1-1154 arp1-67) & MATa $f k s 1: \because H I S 3$, fks2::LYS2, ade3::fks 1-1154:TRP1, arp1::arp1-67:URA3 \\
\hline YOC3073 (fks 1-1154 arp1-73) & MATa $f k s 1: \because H I S 3$, fks $2:: L Y S 2$, ade3::fks 1-1154:TRP1, arp1::arp1-73:URA3 \\
\hline YOC3074 (fks 1-1154 arp 1-79) & MATa $f k s 1: \because H I S 3$, fks $2: \because L Y S 2$, ade3::fks 1-1154:TRP1, arp1::arp1-79:URA3 \\
\hline YOC3075 (fks 1-1154 arp 1-84) & MATa $f k s 1: \because H I S 3$, fks $2 \because: L Y S 2$, ade3::fks 1-1154:TRP1, arp1::arp1-84:URA3 \\
\hline YOC3076 (fks 1-1154 arp 1-108) & MATa $f k s 1: \because H I S 3, f k s 2:: L Y S 2$, ade3::fks 1-1154:TRP1, arp $1: \because \operatorname{arp} 1-108:$ URA3 \\
\hline YOC3077 (fks 1-1154 arp1-122) & MATa $f k s 1:: H I S 3$, fks $2 \because: L Y S 2$, ade3::fks 1-1154:TRP1, arp1::arp1-122:URA3 \\
\hline YOC3078 (fks 1-1154 arp1-133) & MATa $f k s 1: \because H I S 3, f k s 2: \because L Y S 2$, ade3::fks 1-1154:TRP1, arp $1: \because \operatorname{arp} 1-133: U R A 3$ \\
\hline
\end{tabular}


Table I. YeAst STRAINS USED IN THIS STUDY (CONTENUE)

\begin{tabular}{|c|c|}
\hline Strains & Genotype \\
\hline YOC3079 (fks 1-1154 arp 1-162) & MATa $f k s 1: \because H I S 3$, fks $2 \because: L Y S 2$, ade3::fks 1-1154:TRP1, arp1::arp1-162:URA3 \\
\hline YOC3080 (fks 1-1154 arp1-185) & MATa $f k s 1: \because H I S 3$, fks $2:: L Y S 2$, ade3::fks 1-1154:TRP1, arp1::arp1-185:URA3 \\
\hline YOC3081 (fks1-1154 arp1-192) & MATa $f k s 1: \because H I S 3, f k s 2:: L Y S 2$, ade $\because \because f k s 1-1154: T R P 1$, arp $1:$ arp 1-192:URA3 \\
\hline YOC3082 (fks1-1154 arp1-202) & MATa $f k s 1:: H I S 3, f k s 2:: L Y S 2$, ade3::fks1-1154:TRP1, arp1::arp1-202:URA3 \\
\hline YOC3083 (fks1-1154 arp1-214) & MATa $f k s 1:: H I S 3, f k s 2:: L Y S 2$, ade3::fks1-1154:TRP1, arp1::arp1-214:URA3 \\
\hline YOC3084 (fks1-1154 arp1-222) & MATa $f k s 1:: H I S 3, f k s 2:: L Y S 2$, ade3::fks1-1154:TRP1, arp1::arp1-222:URA3 \\
\hline YOC3085 (fks 1-1154 arp1-233) & MATa $f k s 1: \because H I S 3$, fks $2:: L Y S 2$, ade3::fks 1-1154:TRP1, arp1::arp1-233:URA3 \\
\hline YOC3086 (fks 1-1154 arp1-236) & MATa $f k s 1: \because H I S 3, f k s 2:: L Y S 2$, ade3::fks1-1154:TRP1, arp $1: \because \operatorname{arp} 1-236: U R A 3$ \\
\hline YOC3087 (fks 1-1154 arp 1-251) & MATa $f k s 1: \because H I S 3$, fks $2 \because: L Y S 2$, ade3::fks 1-1154:TRP1, arp1::arp1-251:URA3 \\
\hline YOC3088 (fks $1-1154$ arp 1-256) & MATa $f k s 1: \because H I S 3$, fks $2 \because: L Y S 2$, ade3::fks 1-1154:TRP1, arp1::arp1-256:URA3 \\
\hline YOC3089 (fks 1-1154 arp1-263) & MATa $f k s 1: \because H I S 3$, fks $2: \because L Y S 2$, ade3::fks 1-1154:TRP1, arp1::arp1-263:URA3 \\
\hline YOC3090 (fks 1-1154 arp 1-266) & MATa $f k s 1:: H I S 3, f k s 2:: L Y S 2$, ade3::fks1-1154:TRP1, arp1::arp1-266:URA3 \\
\hline YOC3091 (fks 1-1154 arp1-282) & MATa $f k s 1: \because H I S 3, f k s 2: \because L Y S 2$, ade3::fks1-1154:TRP1, arp $1: \because \operatorname{arp} 1-282: U R A 3$ \\
\hline YOC3092 (fks1-1154 arp1-294) & MATa $f k s 1:: H I S 3, f k s 2:: L Y S 2$, ade3::fks1-1154:TRP1, arp1::arp1-294:URA3 \\
\hline YOC3093 (fks1-1154 arp1-300) & MATa $f k s 1:: H I S 3, f k s 2:: L Y S 2$, ade3::fks1-1154:TRP1, arp1::arp1-300:URA3 \\
\hline YOC3094 (fks 1-1154 arp1-317) & MATa $f k s 1: \because H I S 3, f k s 2:: L Y S 2$, ade3::fks1-1154:TRP1, arp $1: \because \operatorname{arp} 1-317:$ URA3 \\
\hline YOC3095 (fks 1-1154 arp1-326) & MATa $f k s 1: \because H I S 3, f k s 2:: L Y S 2$, ade3::fks 1-1154:TRP1, arp $1: \because \operatorname{arp} 1-326: U R A 3$ \\
\hline YOC3096 (fks $1-1154$ arp $1-336)$ & MATa $f k s 1: \because H I S 3, f k s 2:: L Y S 2$, ade3::fks1-1154:TRP1, arp1::arp1-336:URA3 \\
\hline YOC3097 (fks 1-1154 arp1-344) & MATa $f k s 1: \because H I S 3, f k s 2: \because L Y S 2$, ade3::fks1-1154:TRP1, arp1::arp1-344:URA3 \\
\hline YOC3098 (fks $1-1154 \operatorname{arp} 1-368)$ & MATa $f k s 1: \because H I S 3$, fks $2 \because: L Y S 2$, ade3::fks 1-1154:TRP1, arp1::arp1-368:URA3 \\
\hline YOC3099 (fks 1-1154 arp1-374) & MATa $f k s 1: \because H I S 3, f k s 2: \because L Y S 2$, ade3::fks1-1154:TRP1, arp $1:$ arp 1-374:URA3 \\
\hline YOC4106 & MATa $f k s 1: \because H I S 3$, fks2::LYS2, ade3::FKS1:TRP1 kar $9: \because C g-L E U 2$ [pYO2763] \\
\hline YOC4107 & MATa fks $1:: H I S 3$, fks $2:: L Y S 2$, ade $3:$ FKS1:TRP1, wac1, kar9::Cg-LEU2 [pYO2763] \\
\hline YOC4108 & MATa $f k s 1: \because H I S 3$, fks $2:: L Y S 2$, ade $3:: F K S 1: T R P 1$, arp $1: \because \operatorname{KanMX}, \operatorname{kar} 9: \because C g-L E U 2$ [pYO2763] \\
\hline YOC4109 & MATa $f k s 1:: H I S 3, f k s 2:: L Y S 2$, ade3::FKS1:TRP1, arp $1:$ arp 1-31:URA3, kar9::Cg-LEU2 [pYO2763] \\
\hline YOC4110 & MATa $f k s 1:: H I S 3, f k s 2:: L Y S 2$, ade3::FKS1:TRP1, arp1::arp1-236:URA3, kar9::Cg-LEU2 [pYO2763] \\
\hline YOC4111 & MATa $f k s 1: \because H I S 3, f k s 2: \because L Y S 2$, ade3::FKS1:TRP1, arp1::arp1-266:URA3, kar9::Cg-LEU2 [pYO2763] \\
\hline YOC4112 & MATa $f k s 1: \because H I S 3, f k s 2:: L Y S 2$, ade3::FKS1:TRP1, arp $1: \because \operatorname{arp} 1-374: U R A 3$, kar9 $\because$ Cg-LEU2 [pYO2763] \\
\hline
\end{tabular}

* Cg-LEU2 indicates Candida glablata LEU2 gene.

\section{Examination of the cell wall integrity checkpoint defect}

Cells were cultured to a density of $10^{7}$ cells $/ \mathrm{ml}$ at $25^{\circ} \mathrm{C}$ in YPD medium, and $100 \mathrm{ml}$ of cells were centrifuged and resuspended in $10 \mathrm{ml}$ of aqueous medium. The cells were sonicated and loaded into an elutriator (CR20E centrifuge with R5E rotor, Hitachi, Tokyo, Japan). Centrifugation was carried out at $870 \times g$ at $4^{\circ} \mathrm{C}$. The flow rate was adjusted to $10 \mathrm{ml} / \mathrm{min}$ and was shifted to $12 \mathrm{ml} /$ min before collecting $200 \mathrm{ml}$ of G1 cells to be used for synchronized culture. The collected cells were concentrated by centrifugation, resuspended in fresh medium, and incubated at the restrictive temperature $\left(37^{\circ} \mathrm{C}\right)$. Cells were collected every $30 \mathrm{~min}$ and fixed with $3.7 \%$ formaldehyde. The fixed cells were washed three times with SP buffer $(0.5 \mathrm{mg} / \mathrm{ml} \mathrm{BSA,} 150 \mathrm{mM} \mathrm{NaCl}, 50 \mathrm{mM}$ HEPES, $0.1 \%$ Tween-20, and $1 \mathrm{mM} \mathrm{NaN}_{3}$ at $\left.\mathrm{pH} 7.5\right)$ and treated with 28 $\mu \mathrm{g} / \mathrm{ml}$ zymolyase 100T (Seikagaku Corporation, Tokyo, Japan), for $30 \mathrm{~min}$ at $30^{\circ} \mathrm{C}$. The cells were washed with SP buffer and incubated in SP buffer containing $0.1 \%$ Triton X-100 for $10 \mathrm{~min}$ at room temperature. The cells were then washed twice and suspended in an appropriate volume of SP buffer. To visualize spindles, the cells were spread on a poly-L-lysine-coated slide and stained with rat monoclonal anti-yeast-tubulin antibody (1/34 YOL;
Oxford Biotechnology, Oxford, UK) and Alexa488-conjugated goat-anti-rat antibody (Molecular Probes, Eugene, OR) as first and second antibodies, respectively. After the antibody treatment, the cells were mounted in mounting buffer $(90 \%$ glycerol containing 1 $\mathrm{mg} / \mathrm{ml}$ p-phenylenediamine) containing $200 \mathrm{ng} / \mathrm{ml}$ DAPI, overlaid with a coverslip, and sealed. The stained cells were observed and photographed with a fluorescence microscope (DMRE, Leica, Bannockburn, IL) using a $100 \times$ objective. At least 200 cells were counted.

\section{Examination of the nuclear migration defect}

Cells were cultured to mid-log phase in YPD medium at $25^{\circ} \mathrm{C}$. Synchronized G1 cells prepared by centrifugal elutriation were incubated in YPD medium at $37^{\circ} \mathrm{C}$ for $4 \mathrm{~h}$; cells were collected every $30 \mathrm{~min}$ and fixed in $3.7 \%$ formaldehyde. The fixed cells were stained with DAPI and observed with a DMRE microscope (Leica). Only cells that had completed nuclear division were counted, and the percentage of cells with two nuclei in the mother cell after the first cell cycle was determined. At least 200 cells were counted. 
Table II. Plasmids USED IN THIS STUDY

\begin{tabular}{|c|c|}
\hline Name & Description \\
\hline pYO2521 (pRS315-ARP1) & $L E U 2, A R P 1, C E N$ \\
\hline pRS316 & URA3, CEN \\
\hline pBluescript II SK(+) & Used for constructing plasmids for integration \\
\hline pEGFP & $E G F P$ \\
\hline pBS-Cg-LEU2 & Candida glablata LEU2 \\
\hline pYO2522 (pBS-ARP1-URA3) & Used for integrating $A R P 1: U R A 3$ in the $A R P 1$ locus \\
\hline pYO2523 (pBS-wac1-URA3) & Used for integrating wacl:URA3 in the $A R P 1$ locus \\
\hline pYO2524 (pBS-arp1-2-URA3) & Used for integrating arp 1-2:URA3 in the $A R P 1$ locus \\
\hline pYO2526 (pBS-arp1-31-URA3) & Used for integrating arp1-31:URA3 in the $A R P 1$ locus \\
\hline pYO2526 (pBS-arp1-35-URA3) & Used for integrating arp 1-35:URA3 in the $A R P 1$ locus \\
\hline pYO2527 (pBS-arp1-46-URA3) & Used for integrating arp1-46:URA3 in the $A R P 1$ locus \\
\hline pYO2528 (pBS-arp1-67-URA3) & Used for integrating arp 1-67:URA3 in the $A R P 1$ locus \\
\hline pYO2529 (pBS-arp1-73-URA3) & Used for integrating arp 1-73:URA3 in the $A R P 1$ locus \\
\hline pYO2530 (pBS-arp1-79-URA3) & Used for integrating arp1-79:URA3 in the $A R P 1$ locus \\
\hline pYO2531 (pBS-arp1-84-URA3) & Used for integrating arp1-84:URA3 in the $A R P 1$ locus \\
\hline pYO2532 (pBS-arp1-108-URA3) & Used for integrating arp 1-108:URA3 in the $A R P 1$ locus \\
\hline pYO2533 (pBS-arp1-122-URA3) & Used for integrating arp 1-122:URA3 in the $A R P 1$ locus \\
\hline pYO2534 (pBS-arp1-133-URA3) & Used for integrating arp1-133:URA3 in the $A R P 1$ locus \\
\hline pYO2535 (pBS-arp1-162-URA3) & Used for integrating arp 1-162:URA3 in the $A R P 1$ locus \\
\hline pYO2536 (pBS-arp1-185-URA3) & Used for integrating arp1-185:URA3 in the $A R P 1$ locus \\
\hline pYO2537 (pBS-arp1-192-URA3) & Used for integrating arp1-192:URA3 in the $A R P 1$ locus \\
\hline pYO2538 (pBS-arp1-202-URA3) & Used for integrating arp 1-202:URA3 in the $A R P 1$ locus \\
\hline pYO2539 (pBS-arp1-214-URA3) & Used for integrating arp 1-214:URA3 in the $A R P 1$ locus \\
\hline pYO2540 (pBS-arp1-222-URA3) & Used for integrating arp 1-222:URA3 in the $A R P 1$ locus \\
\hline pYO2541 (pBS-arp1-233-URA3) & Used for integrating arp 1-233:URA3 in the $A R P 1$ locus \\
\hline pYO2542 (pBS-arp1-236-URA3) & Used for integrating arp1-236:URA3 in the $A R P 1$ locus \\
\hline pYO2543 (pBS-arp1-251-URA3) & Used for integrating arp 1-251:URA3 in the $A R P 1$ locus \\
\hline pYO2544 (pBS-arp1-256-URA3) & Used for integrating arp1-256:URA3 in the $A R P 1$ locus \\
\hline pYO2545 (pBS-arp1-263-URA3) & Used for integrating arp 1-263:URA3 in the $A R P 1$ locus \\
\hline pYO2546 (pBS-arp1-266-URA3) & Used for integrating arp1-266:URA3 in the $A R P 1$ locus \\
\hline pYO2547 (pBS-arp1-282-URA3) & Used for integrating arp 1-282:URA3 in the $A R P 1$ locus \\
\hline pYO2548 (pBS-arp1-294-URA3) & Used for integrating arp1-294:URA3 in the $A R P 1$ locus \\
\hline pYO2549 (pBS-arp1-300-URA3) & Used for integrating arp 1-300:URA3 in the $A R P 1$ locus \\
\hline pYO2550 (pBS-arp1-317-URA3) & Used for integrating arp1-317:URA3 in the $A R P 1$ locus \\
\hline pYO2551 (pBS-arp1-326-URA3) & Used for integrating arp1-326:URA3 in the $A R P 1$ locus \\
\hline pYO2552 (pBS-arp1-336-URA3) & Used for integrating arp1-336:URA3 in the $A R P 1$ locus \\
\hline pYO2553 (pBS-arp1-344-URA3) & Used for integrating arp 1-344:URA3 in the $A R P 1$ locus \\
\hline pYO2554 (pBS-arp1-368-URA3) & Used for integrating arp1-368:URA3 in the $A R P 1$ locus \\
\hline pYO2555 (pBS-arp1-374-URA3) & Used for integrating arp1-374:URA3 in the $A R P 1$ locus \\
\hline pYO2763 (pRS316-KAR9-URA3) & Used for testing the synthetic lethality between $\operatorname{arp} 1-x$ and $\Delta k a r 9$ \\
\hline
\end{tabular}




\section{Results and Discussion}

\section{Construction of ARP1 charged cluster-to-alanine alleles}

To investigate the structure-function relationship of Arplp, we used a comprehensive mutagenesis method called "charged cluster-to-alanine scanning mutagenesis," in which a cluster of charged amino acids in a target protein is replaced with alanines. In this procedure, it is expected that the surfaces of functional domains are altered with minimal misfolding or structural alterations at a distance from the mutated site. The algorithm for selecting the target clusters was two or three charged amino acids (Glu, Asp, Arg, Lys, or His) in a window of five. As a result, 32 clusters of charged amino acids were identified in the amino acid sequence of Arp1p (Fig. 1). Using a PCR-based mutagenesis method, we constructed a series of mutated arp 1 alleles (designated $\operatorname{arp} 1-x$, where $x$ indicates the number of the first

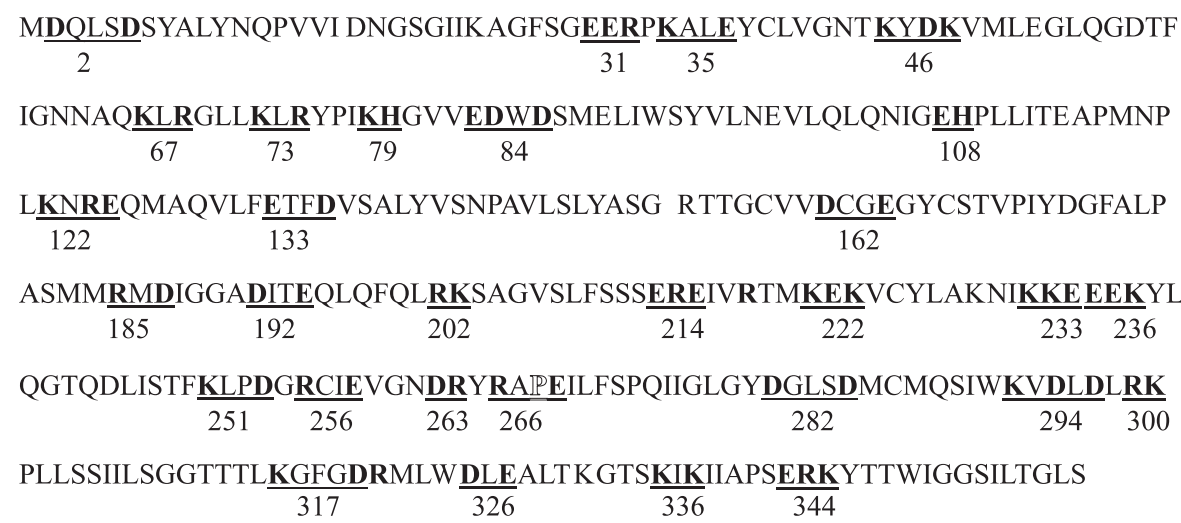

TFQRLWT KKSDWLEDSTRVYSNLM

$\frac{1}{368} \quad \frac{374}{374}$

Fig. 1. Amino acid sequence of the yeast Arp1p. Charged clusters are underlined (with allele numbers below), and the residues that are replaced with alanine are in boldface. A previously identified checkpoint-defective mutation is shown with a white letter: wacl, P268L.

(2)
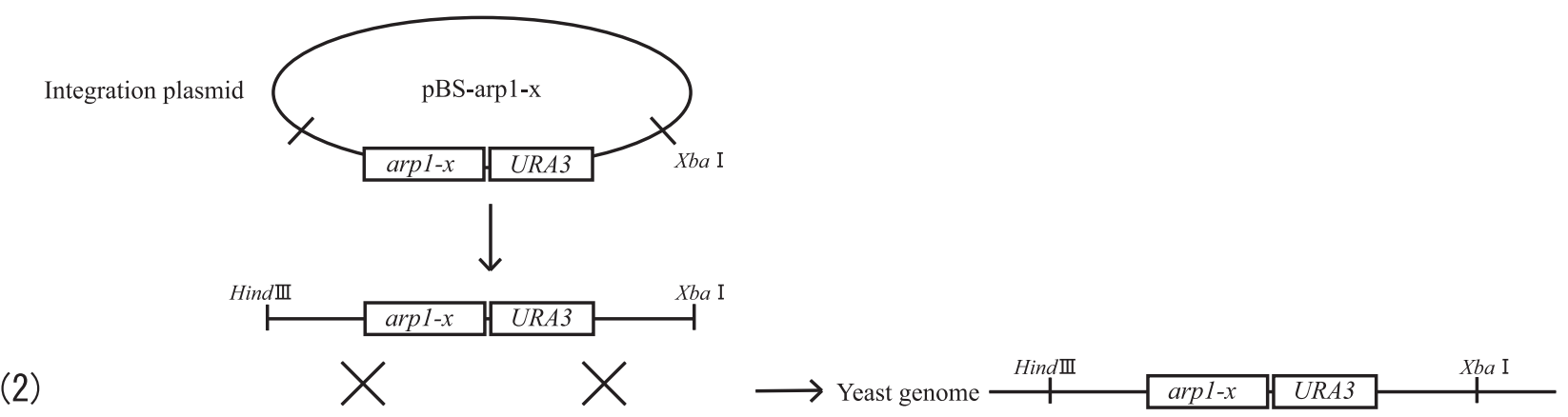

Integration plasmid

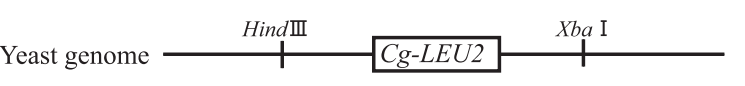

(1)

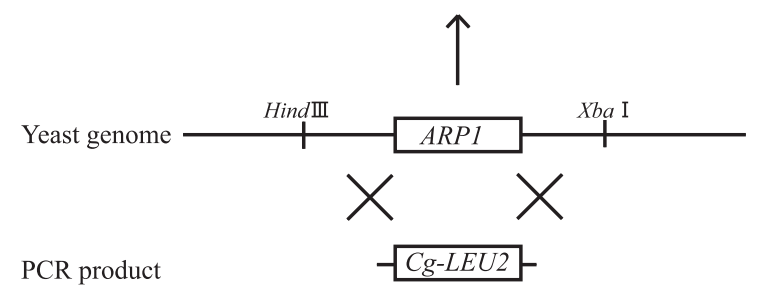

Fig. 2. Diagram of the strategy for integrating the $U R A 3$-linked $\operatorname{arp} 1-x$ allele into the yeast genome at the $A R P 1$ locus. (1) Genomic $A R P 1$ was deleted via homologous recombination with a PCR product composed of $C g-L E U 2$ with 45 bp of the sequence flanking the coding region. (2) Homologous recombination between the HindIII-XbaI fragment of pBS-arp1-x-URA3 and the chromosome containing a Cg-LEU2 replacement of $A R P 1$ ( $\triangle a r p 1:: L E U 2$ ) results in a strain with $U R A 3$-linked $\operatorname{arp1-x}(\operatorname{arp} 1-x: U R A 3)$ at the $A R P 1$ locus. 
amino acid mutated in a charged cluster) that have alanine mutations at the clusters of charged amino acids, and then integrated the mutated alleles in the yeast chromosome (Fig. 2).

\section{Alleles affecting the cell wall integrity checkpoint}

We examined the effect of alanine mutations in Arp1p on the cell wall integrity checkpoint in the $f k s 1-1154$ arp 1-x cells (YOC3066-YOC3099). Synchronized G1 cells of each strain were prepared by centrifugal elutriation and were incubated in YPD medium at the restrictive temperature $\left(37^{\circ} \mathrm{C}\right)$. Cell cycle progression was monitored at $30-\mathrm{min}$ intervals by observing the assembly of the bipolar spindle. To activate the cell wall integrity checkpoint, we used the fks 1-1154 mutant, a temperature-sensitive mutant defective in 1,3- $\beta$-glucan synthase activity. In a control strain, which contained URA3-linked wacl at the ARP1 locus, about $80 \%$ of the cells assembled bipolar spindles at $180 \mathrm{~min}$ after the temperature shift, as did checkpoint-defective wacl cells (Fig. 3). In nine of the 32 mutant strains ( $f k s 1-1154$ arp 1-73, $-84,-162,-214,-222,-233,-236,-263$, and -266 ), over $50 \%$ of the cells did not arrest at G2 phase and assembled bipolar spindles. The remaining mutant strains exhibited slight or almost no effect on the cell wall integrity checkpoint. These results imply that the amino acids mutated in the arp 1-73, $-84,-162,-214,-222,-233,-236,-263$, and -266 alleles are required for the cell wall integrity checkpoint function of the Arp1p molecule.
(A)

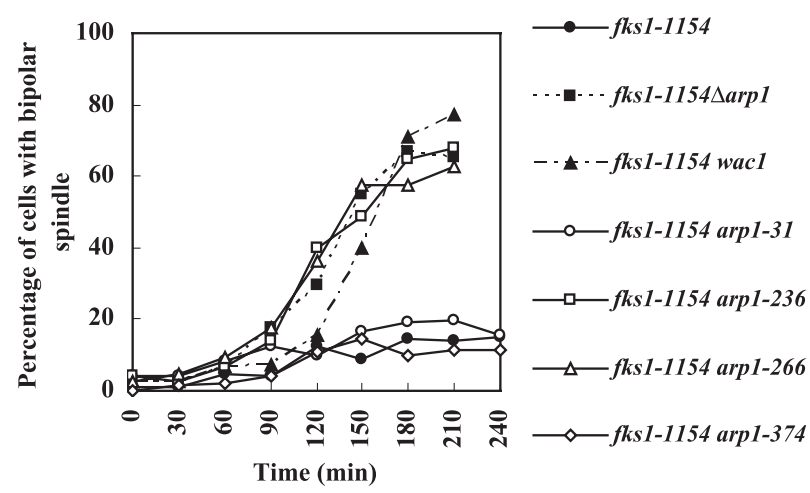

(B)

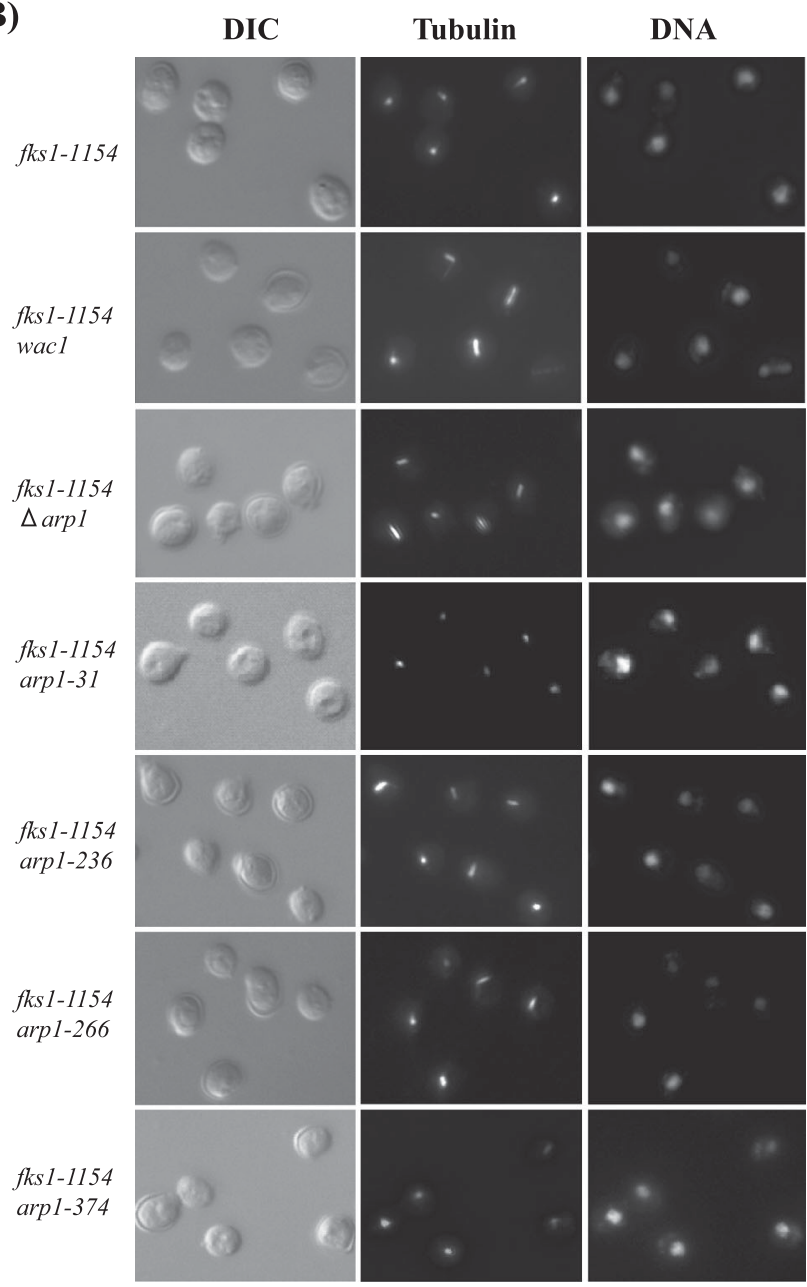

Fig. 3. Effect of the alanine-replaced mutations on the cell wall integrity checkpoint.

(A) Synchronized G1 cells of $f k s 1-1154$ (YOC1087), fks1-1154 wacl (YOC2858), fks1-1154 $\Delta a r p 1$ (YOC2889), fks 1-1154 arp1-31 (YOC3069), fks 1-1154 arp1-236 (YOC3086), fks 1-1154 arp1-266 (YOC3090), and fks 1-1154 arp1-374 (YOC3099) were prepared by centrifugal elutriation and then incubated at the restrictive temperature $\left(37^{\circ} \mathrm{C}\right)$. The percentage of cells with bipolar spindles was examined every $30 \mathrm{~min}$ after the temperature shift. At least 200 cells were counted. (B) Fluorescence microscopy of tubulin, the spindle polar body, and the nucleus. Photographs were taken at 180 min after the temperature shift. 


\section{Alleles affecting nuclear migration}

We examined the effect of alanine mutations in Arplp on nuclear migration by observing the accumulation of cells that did not segregate a nucleus into the daughter cell in FKS1 arp1-x cells (YOC3032-YOC3065). In 14 of the 32 mutant strains (FKS1 arp 1-46, -73, -84, -108, -162, -214, $-222,-251,-266,-294,-326,-344,-368$, and -374$)$, over $20 \%$ of the cells accumulated as mother cells with two nuclei, as in the nuclear migration-defective FKS1 $\triangle a r p 1$ cells (Fig. 4). In the remaining mutant strains, including the wacl mutant, most of cells properly segregated one nucleus into each daughter cell (Fig. 4). These results suggest that the amino acids mutated in arp 1-46, -73, -84, -108, -162, $-214,-222,-251,-266,-294,-326,-344,-368$, and -374 are required for the nuclear migration function of the Arp1p molecule.

Most of the arp1-x alleles could be classified into one of four groups based on these phenotypes (Table III). The first group of mutants (arp1-2, -31, -35, -79, -133, -185, -192, $-202,-256,-282,-300$, and -317 ) shows almost no difference from wild type. The second group of mutants (arp1-
(A)

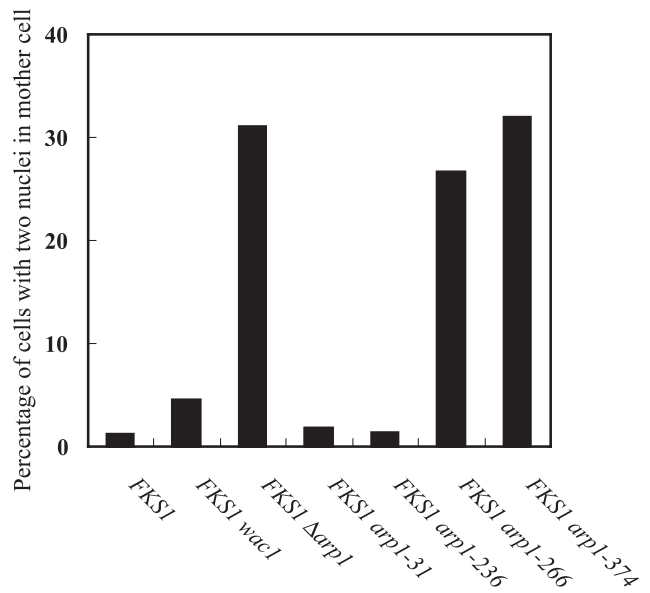

(C)
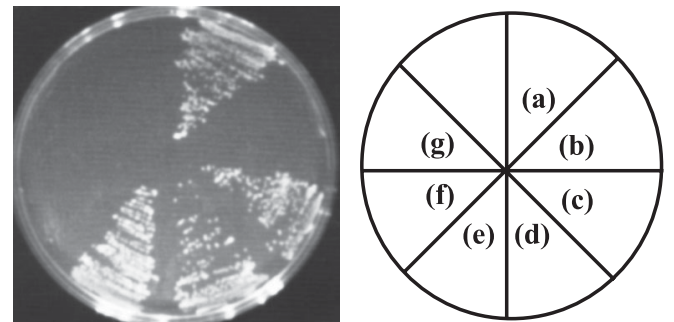

(a) YOC4106 (FKS1 ARP1 $\Delta k a r 9$ [YCp-KAR9::URA3])

(b) YOC4107 (FKS1 $\Delta$ arpl 1 kar9 [YCp-KAR9::URA3])

(c) YOC4108 (FKS1 wac1 $\Delta$ kar9 [YCp-KAR9::URA3])

(d) YOC4109 (FKS1 arp1-31 1 kar9 [YCp-KAR9::URA3])

(e) YOC4110 (FKS1 arp 1-236 $\Delta$ kar9 [YCp-KAR9::URA3])

(f) YOC4111 (FKS1 arp1-266 $\Delta$ kar9 [YCp-KAR9::URA3])

(g) YOC4112 (FKS1 arpl-374 $\Delta$ kar9 [YCp-KAR9::URA3])
(B)

FKS1

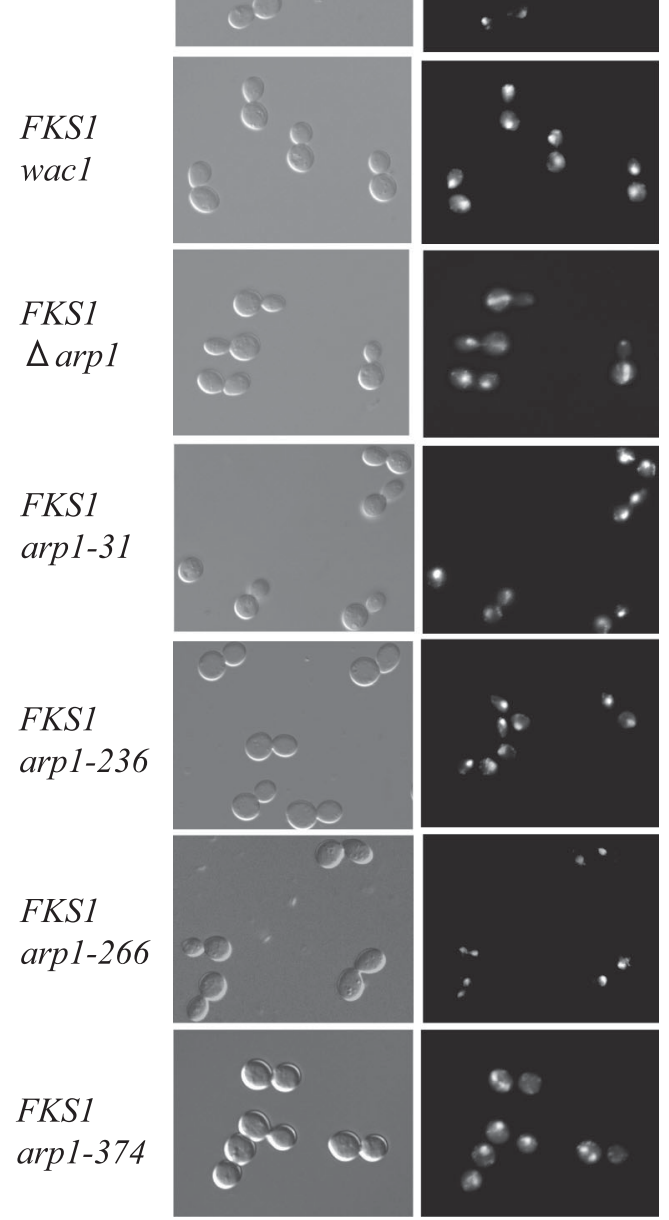

Fig. 4. Effect of the alanine-replaced mutations on nuclear migration.

(A) Synchronized G1 cells of FKS1 ARP1:URA3 (YOC3032), FKS1 wac1:URA3 (YOC3033), FKS1 $\Delta$ arp1 (YOC2888), FKS1 arp1-31 (YOC3035), FKS1 $\operatorname{arp1-236}$ (YOC3052), FKS1 arp 1-266 (YOC3056), and FKS1 arp1-374 (YOC3065) were prepared by centrifugal elutriation and then incubated at $37^{\circ} \mathrm{C}$. The percentage of cells with two nuclei in the mother cell was examined after anaphase. At least 200 cells that had completed nuclear division were counted. (B) Fluorescence microscopy of the nucleus. Photographs were taken at the time when most of the cells had completed nuclear division. (C) The indicated cells were streaked on a plate containing $1 \mu \mathrm{g} / \mathrm{ml} 5$-FOA and were incubated for 2 days at $25^{\circ} \mathrm{C}$. 
TABLE III. EXPERIMENTAL SUMMARY

\begin{tabular}{|c|c|c|c|}
\hline $\operatorname{arp} 1-x$ alleles & Amino acid replacement & Spindle formation $(\%)^{\mathrm{a})}$ & Cells with two nuclei in mother cell $(\%)^{\mathrm{b})}$ \\
\hline$A R P 1$ & None & 14.5 & 1.2 \\
\hline wacl & $\mathrm{P} 268 \mathrm{~L}$ & 70.1 & 4.5 \\
\hline$\Delta a r p 1$ & Deleted & 72.1 & 30.2 \\
\hline $\operatorname{arpl-2}$ & D2A, D6A & 12.4 & 1.2 \\
\hline $\operatorname{arpl} 1-31$ & E31A, E32A, R33A & 19.3 & 1.8 \\
\hline $\operatorname{arp} 1-35$ & $\mathrm{~K} 35 \mathrm{~A}, \mathrm{E} 38 \mathrm{~A}$ & 11.0 & 2.2 \\
\hline $\operatorname{arp} 1-46$ & K46A, D48A, K49A & 19.6 & 28.9 \\
\hline $\operatorname{arpl-67}$ & K67A, R69A & 35.4 & 13.7 \\
\hline $\operatorname{arp} 1-73$ & $\mathrm{~K} 73 \mathrm{~A}, \mathrm{R} 75 \mathrm{~A}$ & 62.2 & 3.0 \\
\hline $\operatorname{arpl-79}$ & K79A, H80A & 19.5 & 25.1 \\
\hline $\operatorname{arpl} 1-84$ & E84A, D85A, D87A & 54.3 & 31.3 \\
\hline $\operatorname{arpl-108}$ & E108A, H109A & 30.2 & 28.6 \\
\hline $\operatorname{arp} 1-122$ & K122A, R124A, E125A & 11.3 & 9.8 \\
\hline $\operatorname{arp1-133}$ & E133A, D136A & 13.6 & 2.0 \\
\hline $\operatorname{arpl} 1-162$ & D162A, E165A & 57.9 & 29.8 \\
\hline $\operatorname{arp} 1-185$ & R185A, D187A & 12.3 & 3.4 \\
\hline $\operatorname{arpl-192}$ & D192A, E195A & 11.9 & 1.0 \\
\hline $\operatorname{arp1-202}$ & R202A, K203A & 11.1 & 2.7 \\
\hline $\operatorname{arpl}-214$ & E214A, R215A, E216A & 50.1 & 28.8 \\
\hline $\operatorname{arpl}-222$ & K222A, E223A, K224A & 51.7 & 25.4 \\
\hline $\operatorname{arp1-233}$ & K233A,K234A, E235A & 63.3 & 2.2 \\
\hline $\operatorname{arp1-236}$ & E236A, E237A, K238A & 64.7 & 1.4 \\
\hline $\operatorname{arpl} 1-251$ & K251A, D254A & 11.2 & 23.7 \\
\hline $\operatorname{arp} 1-256$ & $\mathrm{R} 256 \mathrm{~A}, \mathrm{E} 259 \mathrm{~A}$ & 17.1 & 4.5 \\
\hline $\operatorname{arp} 1-263$ & D263A, R264A & 58.6 & 2.6 \\
\hline $\operatorname{arp1-266}$ & R266A, E269A & $\mathbf{5 7 . 4}$ & 25.1 \\
\hline $\operatorname{arpl}-282$ & D282A, D286A & 14.0 & 1.6 \\
\hline $\operatorname{arp1-294}$ & K294A, D296A, D298A & 13.6 & 25.0 \\
\hline $\operatorname{arp} 1-300$ & R300A, K301A & 15.8 & 2.5 \\
\hline $\operatorname{arp} 1-317$ & K317A, D321A & 10.0 & 1.5 \\
\hline arp1-326 & D326A, E328A & 12.3 & 30.5 \\
\hline $\operatorname{arpl} 1-336$ & $\mathrm{~K} 336 \mathrm{~A}, \mathrm{~K} 338 \mathrm{~A}$ & 15.5 & 12.1 \\
\hline $\operatorname{arp1}-344$ & E344A, R345A, K346A & 8.3 & 30.2 \\
\hline $\operatorname{arpl} 1-368$ & K368A, K369A, D371A & 8.5 & 27.1 \\
\hline $\operatorname{arp1-374}$ & E374A, D375A, R378A & 10.1 & 31.9 \\
\hline
\end{tabular}

a) Bipolar spindle formation (percentage) was measured in the fks 1-1154 background. Strain: YOC2889 and YOC3066-3099.

b) Cells with two nuclei in mother cell (percentage) was measured in FKS1 background. Strain: YOC2888 and YOC3034-3065.

Mean values of at least two independent experiments are presented. Values judged to be defective in checkpoint or nuclear migration are represented in boldface.

$233,-236$, and -263 ) has a defect only in the cell wall integrity checkpoint and shows an intact nuclear migration function, as do cells with the wacl allele. The third mutant group (arp1-46, -251, -294, -326, -344, -368, and -374) has a defect only in nuclear migration and has an intact cell wall integrity checkpoint function. The fourth group of mutants (arp1-73, -84, -162, -214, -222, and -266) is defective in both cell wall integrity checkpoint and nuclear migration functions, as is the deletion mutant of $A R P 1$.

\section{Alleles affecting the genetic interaction with kar9}

$K A R 9$ is a component of the kinesin-mediated nuclear migration pathway. A mutation in KAR9 can cause a nuclear migration defect that conveys synthetic lethality in conjunction with mutations in genes of the dynein/dynactin pathway (Miller and Rose, 1998). To confirm whether mutants representative of each of the four mutant groups (arp1-31, $-236,-266$, and -374) have defects in dynein/dynactinmediated nuclear migration, we tested for synthetic lethality between arp 1 mutants and $\Delta k a r 9$. Cells of the strains 
YOC4106, YOC4106, YOC4108, YOC4109, YOC4110, YOC4111, and YOC4112 were streaked on a plate containing $1 \mu \mathrm{g} / \mathrm{ml} 5$-FOA and were incubated for 2 days at $25^{\circ} \mathrm{C}$. The mutation combinations of $\Delta$ arpl $\Delta k a r 9$, arpl$266 \Delta k a r 9$, and arp1-374 $\Delta k a r 9$ showed obvious growth defects, whereas the ARP1 $\Delta k a r 9$, wacl $\Delta k a r 9$, arpl-31 $\Delta k a r 9$, and arp1-236 $\Delta$ kar9 strains formed colonies (Fig. 4C). These results further indicate that strains containing arp 1-266 and arp 1-374 have defects in dynein/dynactinmediated nuclear migration, and that strains containing wacl, arp1-31, and arp1-236 do not.

\section{A structural model for the interpretation of Arp1p mutations}

To clarify the structure-function relationship of the Arplp molecule, the three-dimensional structure of the Arplp monomer was predicted using the homology modeling software MODELLER (Marti-Renom et al., 2000; Sali and Blundell, 1993). The prediction was performed with the three-dimensional structure of yeast conventional actin as a template, because Arp $1 \mathrm{p}$ has about 50\% amino acid identity and $70 \%$ similarity with yeast conventional actin and is considered to share a conserved ultrastructure (Goodson and Hawse, 2002; Kabsch and Holmes, 1995; Poch and Winsor, 1997). The putative three-dimensional structure of Arp1p was visualized with RasMol software (Sayle and MilnerWhite, 1995), and the positions of the corresponding alanine-replaced mutations were labeled on the structure (Fig. 5A and B). The amino acids labeled in red are essential only for the cell wall integrity checkpoint function (arp 1-233, -236, -263, and wacl). The positions of these amino acids are concentrated in one domain and are on the surface of the Arp1p molecule, with the exception of the wacl mutation, which is buried in the core of the structure. The amino acids essential for only nuclear migration are shown in green (arp1-46, -251, -294, -326, -344, -368, and -374) and are located on the overall surface of the Arp1p molecule. The positions of the mutations suggest that these residues exposed on the surface of the molecule may be required for physical interactions with Arp1p binding proteins. These physical interactions may determine the functional specificity of Arp $1 p$ related to the cell wall integrity checkpoint or nuclear migration. The amino acids colored yellow are essential for both the cell wall integrity checkpoint and nuclear migration functions (arp 1-73, -84, -162, $-214,-222$, and -266). Several of these residues are concentrated close to the ATP binding cleft that is required for Arp1p monomer polymerization (Bingham and Schroer, 1999). Therefore, the mutation of these amino acids may inhibit Arp1p polymerization, resulting in the destruction of the dynactin complex.

Arp1p forms an actin-like short filament composed of nine or ten Arp1p monomers (Bingham and Schroer, 1999; Schafer et al., 1994). Therefore, the ultrastructure of the
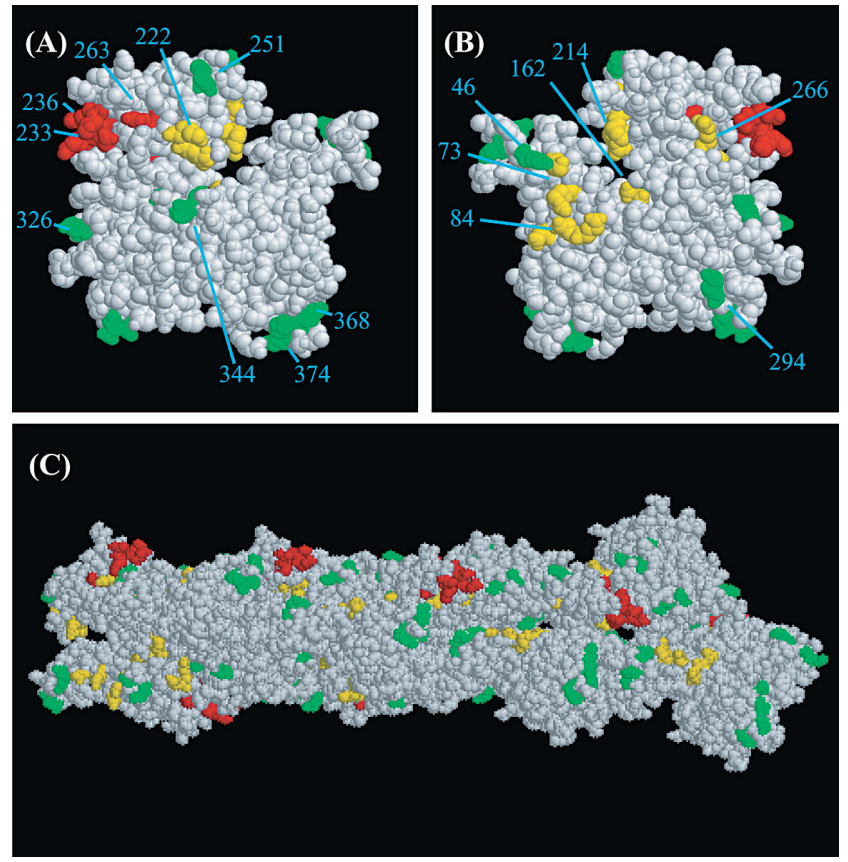

Fig. 5. Positions of the functional residues on the monomer and polymer forms of Act1p.

(A) The three-dimensional structure of the yeast Act1p structure is presented with RasMol molecular visualization software (Sayle and Milner-White, 1995). This view is designated as the "front view" of Actlp. The alanine mutations associated with the different effects are indicated according to the following color scheme: red, cell wall integrity checkpoint defective (over 50\% cells formed bipolar spindle at $180 \mathrm{~min}$ ); green, nuclear migration defective (over $20 \%$ cells have two nuclei in mother cell); and yellow, both cell wall integrity checkpoint and nuclear migration defective. Alleles are represented by number. (B) Same as A, except back view. (C) Ultrastructure of the rabbit muscle actin 8-mer (Lorenz et al., 1993). The colored residues correspond to the equivalent residues in yeast Actlp. Color scheme is the same as A and B.

rabbit muscle actin 8-mer was used for predicting the positions of the mutations on the filamentous form of Arp1p (Fig. 5C) (Lorenz et al., 1993). Most of the amino acids colored red and green are located on the surface of the Arp1p polymer. Thus, mutations in these amino acids are unlikely to inhibit the formation of the Arp1p filament. On the other hand, the amino acids colored yellow tend to lie on the hidden surface of the filament. This supports the possibility that mutations of these residues may cause the destruction of the Arp1p filament, thereby abolishing both the cell wall integrity checkpoint and dynein-mediated nuclear migration functions in these cells. Alternatively, these mutated Arp1p molecules may be rapidly degraded, producing the same phenotype as that of the arpl deletion.

Recently, Clark and Rose (Clark and Rose, 2005) reported that an arp 1 mutant with the same mutations as our arp1214 mutant (E214A, R215A, E216A) has a defect in the polymerization of the Arplp filament. In our study, we 
showed that the arp1-214 mutant is defective in both cell wall integrity checkpoint and nuclear migration. These data suggest that the precise formation of the Arplp filament is essential not only for nuclear migration but also for the cell wall integrity checkpoint. Clark and Rose (Clark and Rose, 2005) proposed that residues K369, D371, E374, and D375 play an important role in the interaction with Jnm1p. We showed that the $\operatorname{arp} 1-368$ (K368A, K369A, D371A) and arp 1-374 (E374A, D375, R378A) mutants were defective only in nuclear migration, suggesting that the interaction of Arp1p with Jnm1p is not critical for its checkpoint function.

In summary, the deletion of arpl causes defects in both cell wall integrity checkpoint and nuclear migration functions, whereas the wacl mutation causes a defect only in the cell wall integrity checkpoint. One possible explanation for the phenotypic difference between wacl and arpl deletion mutants is that the cell wall integrity checkpoint and nuclear migration are based on distinct molecular functions of Arp1p, and that these two functions are separable in the Arp1p molecule. These results, together with our previous results showing that dynein-related proteins, except dynactin subunits, are not required for the cell wall integrity checkpoint, indicate that the molecular function of the cell wall integrity checkpoint may be independent of the dyneinbased nuclear migration mechanism.

Acknowledgments. This work was supported by a grant (\#16026205) for Scientific Research from the Ministry of Education, Science, Sports, and Culture of Japan.

\section{References}

Bingham, J.B. and Schroer, T.A. 1999. Self-regulated polymerization of the actin-related protein Arp1. Curr. Biol., 9: 223-226.

Cid, V.J., Duran, A., del Rey, F., Snyder, M.P., Nombela, C., and Sanchez, M. 1995. Molecular basis of cell integrity and morphogenesis in Saccharomyces cerevisiae. Microbiol. Rev., 59: 345-386.

Clark, S.W. and Rose, M.D. 2005. Alanine scanning of Arp1 delineates a putative binding site for Jnm1/dynamitin and Nip100/p150Glued. Mol. Biol. Cell, 16: 3999-4012.

Eckley, D.M., Gill, S.R., Melkonian, K.A., Bingham, J.B., Goodson, H.V., Heuser, J.E., and Schroer, T.A. 1999. Analysis of dynactin subcomplexes reveals a novel actin-related protein associated with the arp1 minifilament pointed end. J. Cell Biol., 147: 307-320.

Geiser, J.R., Schott, E.J., Kingsbury, T.J., Cole, N.B., Totis, L.J., Bhattacharyya, G., He, L., and Hoyt, M.A. 1997. Saccharomyces cerevisiae genes required in the absence of the CIN8-encoded spindle motor act in functionally diverse mitotic pathways. Mol. Biol. Cell, 8 : $1035-1050$.

Goodson, H.V. and Hawse, W.F. 2002. Molecular evolution of the actin family. J. Cell Sci., 115: 2619-2622.

Hildebrandt, E.R. and Hoyt, M.A. 2000. Mitotic motors in Saccharomyces cerevisiae. Biochim. Biophys. Acta, 1496: 99-116.

Ito, T., Chiba, T., Ozawa, R., Yoshida, M., Hattori, M., and Sakaki, Y. 2001. A comprehensive two-hybrid analysis to explore the yeast protein interactome. Proc. Natl. Acad. Sci. USA, 98: 4569-4574.
Kabsch, W. and Holmes, K.C. 1995. The actin fold. FASEB J., 9: 167174.

Kahana, J.A., Schlenstedt, G., Evanchuk, D.M., Geiser, J.R., Hoyt, M.A., and Silver, P.A. 1998. The yeast dynactin complex is involved in partitioning the mitotic spindle between mother and daughter cells during anaphase B. Mol. Biol. Cell, 9: 1741-1756.

Lew, D.J. and Reed, S.I. 1995. A cell cycle checkpoint monitors cell morphogenesis in budding yeast. J. Cell Biol., 129: 739-749.

Lorenz, M., Popp, D., and Holmes, K.C. 1993. Refinement of the F-actin model against X-ray fiber diffraction data by the use of a directed mutation algorithm. J. Mol. Biol., 234: 826-836.

Marti-Renom, M.A., Stuart, A.C., Fiser, A., Sanchez, R., Melo, F., and Sali, A. 2000. Comparative protein structure modeling of genes and genomes. Annu. Rev. Biophys. Biomol. Struct., 29: 291-325.

McMillan, J.N. and Tatchell, K. 1994. The JNM1 gene in the yeast Saccharomyces cerevisiae is required for nuclear migration and spindle orientation during the mitotic cell cycle. J. Cell Biol., 125: 143-158.

Miller, R.K. and Rose, M.D. 1998. Kar9p is a novel cortical protein required for cytoplasmic microtubule orientation in yeast. J. Cell Biol., 140: $377-390$.

Muhua, L., Karpova, T.S., and Cooper, J.A. 1994. A yeast actin-related protein homologous to that in vertebrate dynactin complex is important for spindle orientation and nuclear migration. Cell, 78: 669-679.

Poch, O. and Winsor, B. 1997 . Who's who among the Saccharomyces cerevisiae actin-related proteins? A classification and nomenclature proposal for a large family. Yeast, 13: 1053-1058.

Rudner, A.D. and Murray, A.W. 1996. The spindle assembly checkpoint. Curr. Opin. Cell Biol., 8: 773-780.

Sali, A. and Blundell, T.L. 1993. Comparative protein modelling by satisfaction of spatial restraints. J. Mol. Biol., 234: 779-815.

Sambrook, J., Fritsch, E.F., and Maniatis, T. 1989. Molecular Cloning. A laboratory manual. Cold Spring Harbor Laboratory Press, Cold Spring Harbor, New York.

Sayle, R.A. and Milner-White, E.J. 1995. RASMOL: biomolecular graphics for all. Trends Biochem. Sci., 20: 374.

Schafer, D.A., Gill, S.R., Cooper, J.A., Heuser, J.E., and Schroer, T.A. 1994. Ultrastructural analysis of the dynactin complex: an actin-related protein is a component of a filament that resembles F-actin. J. Cell Biol., 126: 403-412.

Sherman, F., Fink, G.R., and Hicks J.B. 1986. Methods in Yeast Genetics: A laboratory manual. Cold Spring Harbor Laboratory Press, Cold Spring Harbor, New York.

Sikorski, R.S. and Hieter, P. 1989. A system of shuttle vectors and yeast host strains designed for efficient manipulation of DNA in Saccharomyces cerevisiae. Genetics, 122: 19-27.

Suzuki, M., Igarashi, R., Sekiya, M., Utsugi, T., Morishita, S., Yukawa, M., and Ohya, Y. 2004. Dynactin is involved in a checkpoint to monitor cell wall synthesis in Saccharomyces cerevisiae. Nat. Cell Biol., 6: 861871.

Uetz, P., Giot, L., Cagney, G., Mansfield, T.A., Judson, R.S., Knight, J.R., Lockshon, D., Narayan, V., Srinivasan, M., Pochart, P., Qureshi-Emili, A., Li, Y., Godwin, B., Conover, D., Kalbfleisch, T., Vijayadamodar, G., Yang, M., Johnston, M., Fields, S., and Rothberg, J.M. 2000. A comprehensive analysis of protein-protein interactions in Saccharomyces cerevisiae. Nature, 403: 623-627.

Weinert, T.A., Kiser, G.L., and Hartwell, L.H. 1994. Mitotic checkpoint genes in budding yeast and the dependence of mitosis on DNA replication and repair. Genes Dev., 8: 652-665.

(Received for publication, October 7, 2005 and accepted, October 21, 2005) 